\title{
Inhibiting the proliferation and metastasis of hilar cholangiocarcinoma cells by blocking the expression of vascular endothelial growth factor with small interfering RNA
}

\author{
KE XIAO $^{1}$, ZHENGXIAO OUYANG ${ }^{2}$ and HUI-HUAN TANG ${ }^{3}$ \\ ${ }^{1}$ Department of Gastroduodenal and Pancreatic Surgery, Hunan Cancer Hospital and \\ The Affiliated Cancer Hospital of Xiangya School of Medicine, Central South University, Changsha, Hunan 410013; \\ ${ }^{2}$ Department of Orthopedics, The Second Xiangya Hospital, Central South University, Changsha, Hunan 410011; \\ ${ }^{3}$ Department of General Surgery, Xiangya Hospital, Central South University, Changsha, Hunan 410008, P.R. China
}

Received October 20, 2017; Accepted April 15, 2018

DOI: $10.3892 / \mathrm{ol} .2018 .8840$

\begin{abstract}
The aim of the present study was to investigate whether the proliferation and metastasis of hilar cholangiocarcinoma cells can be suppressed and whether apoptosis can be induced by small interfering RNA (siRNA) repression of vascular endothelial growth factor (VEGF). siRNA sequences targeting the $V E G F$ gene were designed and the human hilar cholangiocarcinoma QBC939, HCCC-9810 and RBE cell lines were transfected with VEGF-siRNA plasmids for $48 \mathrm{~h}$. Reverse transcription-quantitative polymerase chain reaction and western blotting measured the levels of VEGF-A, VEGF-C and matrix metalloproteinase 2 (MMP2) mRNA expression and protein content. The cell invasion potential was evaluated using the Transwell invasion and migration assay and the MTT assay was employed to detect the proliferation of hilar cholangiocarcinoma cells. Flow cytometry was used to quantify cell apoptosis and necrosis. Following the transfection of VEGF-siRNA, a significant reduction of mRNA and protein levels of VEGF-A, VEGF-C and MMP2 was observed in the hilar cholangiocarcinoma cells. The invasion, migration and proliferation of tumor cells were also notably decreased. The rate of tumor cell apoptosis was increased in the VEGF-siRNA group (15.42\%) compared with the non-siRNA control (2.22\%) and the negative control (2.71\%) groups. It was concluded that blocking the expression of VEGF via VEGF-siRNA effectively inhibited the invasion, migration and proliferation, and
\end{abstract}

Correspondence to: Dr Ke Xiao, Department of Gastroduodenal and Pancreatic Surgery, Hunan Cancer Hospital and The Affiliated Cancer Hospital of Xiangya School of Medicine, Central South University, 283 Tongzipo Road, Changsha, Hunan 410013, P.R. China E-mail: anyco_2001@sina.com

Key words: hilar cholangiocarcinoma, small interfering RNA, vascular endothelial growth factor, proliferation, metastasis, apoptosis induced apoptosis in hilar cholangiocarcinoma cells. These observations suggested that targeting VEGF with RNAi may be an effective therapeutic strategy for treating hilar cholangiocarcinoma.

\section{Introduction}

Hilar cholangiocarcinoma $(\mathrm{HC})$ is a primary malignant tumor that originates from the biliary epithelium cholangiocytes and develops at the confluence of the right and left biliary ducts within the portal hepatic duct. HC was first described by Nicolas Klatskin in 1957, and has therefore been termed a 'Klatskin tumor.' These tumors account for $58-75 \%$ of all extrahepatic biliary carcinomas. Patients with Klatskin tumors that receive either conservative treatment or forego surgical_intervention have had a 5-year survival rate $<5 \%$ over the past 50 years. Diagnosis and therapeutic efficacy have also not substantially improved due to the extremely aggressive nature of this neoplasm and its asymptomatic presentation during early stages of illness. Asymptomatic presentation and aggressive growth are associated with a poor prognosis during the advanced stages $(1,2)$. In addition, over the last two decades, the incidence and mortality of hilar cholangiocarcinoma have been increasing worldwide and this has been more significant in the Far East than in the West (3-5).

Recent studies have suggested that neo-angiogenesis serves a key role in the growth and spreading of tumor mass, including in cholangiocarcinoma (6-8). Although numerous growth factors are involved, the vascular endothelial growth factor (VEGF) has been revealed to make an important contribution to the angiogenesis in a solid tumor and its metastasis $(9,10)$. Our recent study demonstrated that expression of VEGF and matrix metalloproteinase-2 (MMP2) was significantly higher in cholangiocarcinoma tissues than in normal bile duct tissues and para-neoplastic tissues (11). Among the VEGF family, VEGF-A has the largest effect in promoting angiogenesis, whereas VEGF-C has recently been revealed to induce angiogenesis and lymphangiogenesis $(12,13)$. 
Recently, small interfering RNA (siRNA) has been revealed to inhibit the expression of a corresponding target gene in mammals through the biologically conserved mechanism of RNA interference (RNAi) $(14,15)$. RNAi is a sequence-specific, post-transcriptional gene-silencing mechanism that allows for specific repression of target genes (16-19). RNAi is now being exploited as a powerful tool for the analysis of gene function and demonstrates potential for therapeutic applications.

There have been a number of studies evaluating VEGF in the treatment of tumors, including gastrointestinal cancer, liver cancer, mammary cancer, prostate cancer and pancreatic cancer (20-24). In addition to these studies, several anti-angiogenic drugs, including Bevacizumab and Celecoxib, have been developed and used in clinical practice $(25,26)$. However, there have been no studies on VEGF in hilar cholangiocarcinomas. The aim of the present study was to identify an improved VEGF-specific RNAi for the preclinical treatment of hilar cholangiocarcinoma.

\section{Materials and methods}

Cell culture. Cell lines used for experimentation were the normal human bile duct epithelial HIBEpiC cell line, in addition to the QBC939, HCCC-9810 and RBE cell lines, which are all poorly differentiated hilar cholangiocarcinoma cells originating from adenocarcinoma. The cell lines were obtained from Third Military Medical University (Chongqing, China), Type Culture Collection of the Chinese Academy of Sciences (Shanghai, China) and American Type Culture Collection (Manassas, VA, USA), respectively. All cell lines were maintained in Dulbecco's modified Eagle's medium (DMEM; Invitrogen; Thermo Fisher Scientific, Inc., Waltham, MA, USA) supplemented with 10\% fetal calf serum (Gibco; Thermo Fisher Scientific, Inc., Waltham, MA, USA) and no antibiotics at $37^{\circ} \mathrm{C}$ in a humidified atmosphere with $5 \% \mathrm{CO}_{2}$. Streptomycin $(100 \mu \mathrm{g} / \mathrm{ml})$ and penicillin $(100 \mathrm{U} / \mathrm{ml})$ were later added to the medium.

The expression of VEGF in hilar cholangiocarcinoma lines. The mRNA expression of VEGF was detected by reverse transcription-quantitative polymerase chain reaction (RT-qPCR). First, total RNA from each experimental group was extracted using TRIzol reagent (Invitrogen; Thermo Fisher Scientific, Inc.) followed by removal of DNA according to the manufacturer's protocols.

The reverse transcription was performed using 20- $\mu 1$ reactions of RevertAid ${ }^{\mathrm{TM}}$ M-MulV Reverse Transcriptase (Fermentas; Thermo Fisher Scientific, Inc., Pittsburgh, PA, USA) according to the manufacturer's protocols.

RT-qPCR was performed using SYBR Green PCR Master mix (Applied Biosystems; Thermo Fisher Scientific, Inc.) with an ABI Prism 7500 Sequence Detection system (Applied Biosystems; Thermo Fisher Scientific, Inc.). The amplification conditions were as follows: Denaturation for $10 \mathrm{sec}$ at $95^{\circ} \mathrm{C}$, 40 amplification cycles $\left(95^{\circ} \mathrm{C} 5 \mathrm{sec}, 60^{\circ} \mathrm{C} 20 \mathrm{sec}\right)$, and generation of a dissociation curve $\left(95^{\circ} \mathrm{C} 60 \mathrm{sec}, 55^{\circ} \mathrm{C} 30 \mathrm{sec}\right)$. The primer sequences were as follows: VEGF, 5'-TGCCCACTG AGGAGTCCAAC-3'; VEGF-A (226 bp) forward, 5'-CGTGTA CGTTGGTGCCCGCT-3' and reverse, 5'-TCCTTCCTCCTG CCCGGCTC-3'; VEGF-C (200 bp) forward, 5'-AGCCAA TGTGGGGCCAACCG-3' and reverse, 5'-TGGCGGTTCGTA
CATGGCCG-3'; MMP2 (235 bp) forward, 5'-GGCAATGCA GTGGGGGCTTAA-3' and reverse, 5'-GCTCTCCTTGGG GCAGCCATAGA-3'; and $\beta$-actin (208 bp) forward, 5'-ACA GAGCCTCGCCTTTGCCGATC-3' and reverse, 5'-ATCCTT CTGACCCATGCCCACCA-3'. The expression of the target gene was measured using the $2^{-\Delta \Delta C q}$ method (27). Experiments were performed in triplicate.

The protein expression of VEGF was detected by western blotting. The primary antibodies used for western blotting were rabbit VEGF-A (catalog no., sc-152; dilution, 1:200; Santa Cruz Biotechnology, Dallas, TX, USA), rabbit VEGF-C (catalog no., sc-25783; dilution, 1:200; Santa Cruz Biotechnology, Dallas, TX, USA), and mouse MMP2 (catalog no., sc-13594; dilution, 1:300; Santa Cruz Biotechnology, Dallas, TX, USA), respectively. A mouse GAPDH antibody (catalog no., Mab-2005079; dilution, 1:8,000; Promab Biotechnologies, Inc., Richmond, CA, USA) was used as an internal standard. The secondary antibody was goat anti rabbit IgG-HRP (catalog no., sc-2004; Santa Cruz Biotechnology, Inc.), with dilution $1: 10,000 ; 1: 10,000 ; 1: 20,000$ and 1:80,000, respectively. All reagents were used according to the manufacturer's protocol.

The cell protein was obtained using the RIPA buffer (Promab Biotechnologies, Inc.) and quantified by the BCA method. Equal amounts of extracted protein were run on an SDS-PAGE gel (12\%) and transferred onto a PVDF membrane. The membranes were blocked with $5 \%$ skimmed dry milk in phosphate-buffered saline (PBS) for $2 \mathrm{~h}$ at $37^{\circ} \mathrm{C}$, followed by incubation with the primary antibodies for $2 \mathrm{~h}$ at room temperature. The membrane was rinsed four times with PBST and then incubated with secondary antibody for $1 \mathrm{~h}$ at room temperature. To remove the secondary antibody, the membrane was washed four times with PBST. The protein was detected with the SuperSignal protein detection kit (Pierce; Thermo Fisher Scientific, Inc.). These data were compared and analyzed by Gel-pro Analyzer 4.0 software (Media Cybernetics, Inc., Rockville, MD, USA) and normalized to GAPDH. The whole experiment was performed in triplicate and results were represented as ratios of VEGF to GAPDH.

VEGF-siRNA preparation and transfection. Three VEGF specific siRNA fragments and one scrambled shRNA (negative control) were synthesized by Shanghai Gene Pharma Co., Ltd. (Shanghai, China), according to the manufacturer's protocols. The two complementary oligonucleotides of VEGF-siRNA were: siRNA1 forward, 5'-GGCCAGCACAUAGGAGAG ATT-3' and reverse, 5'-UCUCUCCUAUGUGCUGGCCTT-3'; siRNA2 forward, 5'-ACCTCACCAAGGCCAGCACTT-3' and reverse, 5'-GTGCTGGCCTTGGTGAGGTTT-3'; siRNA3 forward, 5'-GTGGTGAAGTTCATGGATGTT-3' and reverse, 5'-CATCCATGAACTTCACCACTT-3'; and scrambled shRNA forward, 5'-CACCGTTCTCCGAACGTGTCACGTCAAG AGATTACGTGACACGTTCGGAGAATTTTTTG-3' and reverse, 5'-GATCCAAAAAATTCTCCGAACGTGTCAC GTAATCTCTTGACGTGACACGTTCGGAGAAC-3'. The reconstructed plasmids were verified by sequence analysis at Promab Biotechnologies, Inc. These constructs were named siRNA-VEGF-1, siRNA-VEGF-2, siRNA-VEGF-3 and siRNA-VEGF-NC, respectively.

QBC939, HCCC-9810 and RBE cell lines were transfected with siRNA-VEGF-1, siRNA-VEGF-2, siRNA-VEGF-3 and 

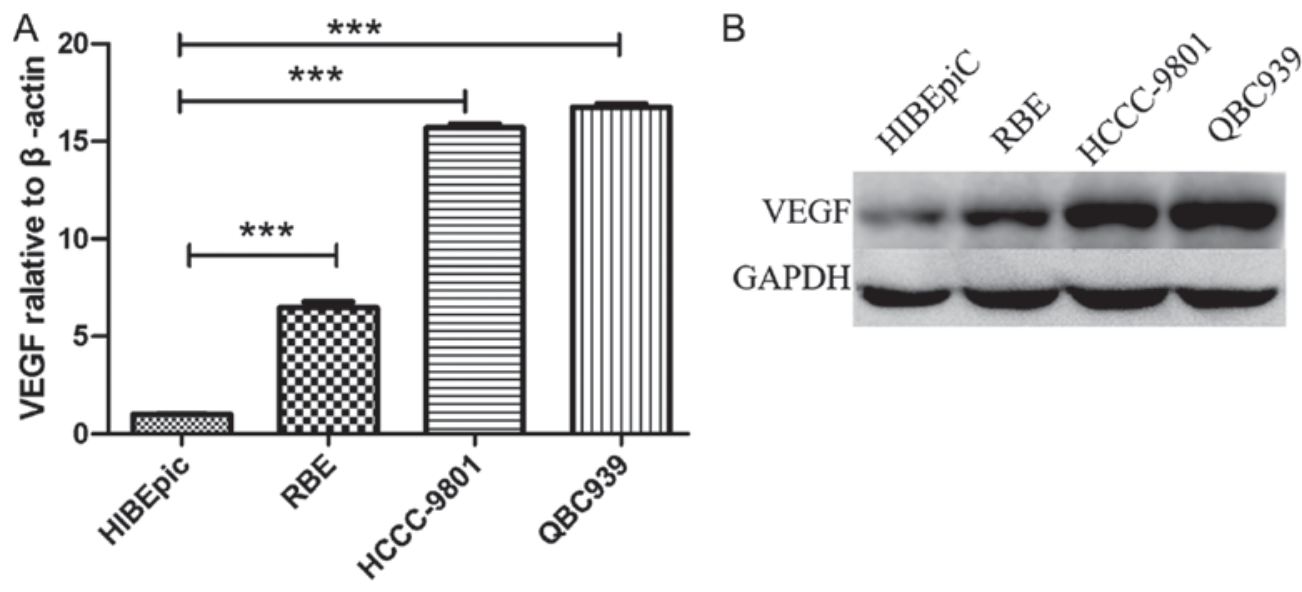

Figure 1. Expression of VEGF in hilar cholangiocarcinoma lines. (A) Quantification and (B) western blot analysis of the expression profile of VEGF in normal human bile duct epithelial cells and hilar cholangiocarcinoma cells assayed by reverse transcription-quantitative polymerase chain reaction and western blotting. The relative level of VEGF normalized to $\beta$-actin in the HIBEpiC group was set to 1 . The normalized VEGF expression levels were as follows: RBE, 6.21 \pm 0.10 ; HCCC-9801, 15.35 \pm 0.23 ; and QBC939, 16.62 \pm 0.34 . $^{* * *} \mathrm{P}<0.001$. VEGF, vascular endothelial growth factor.

shRNA-VEGF-NC using Lipofectamine ${ }^{\mathrm{TM}} 2000$ (Invitrogen; Thermo Fisher Scientific, Inc., Waltham, MA, USA) according to the manufacturer's protocols. In a word, cells were seeded in a six-well plate (Corning Incorporated, USA) at 50-60\% confluence. After cultivation for $24 \mathrm{~h}$, the cells were transfected with $100 \mathrm{nM}$ siRNAs using Lipofectamine 2000. Fluorescence microscopy (at x1,000 magnification) was used to monitor the transfection of each cell following a $6 \mathrm{~h}$ incubation. Following transfection for $48 \mathrm{~h}\left(37^{\circ} \mathrm{C}, 5 \% \mathrm{CO}_{2}\right)$, cells were harvested for additional experiments.

In vitro invasion assay. The invasion assay was performed using Transwell polycarbonate membranes inserted into 6-well plates (Costar; Corning Incorporated, Corning, NY, USA) according to the manufacturer's protocols. Briefly, Transwell filters were coated with Matrigel $(3.9 \mu \mathrm{g} / 1,60-80 \mu \mathrm{l}$; BD Biosciences, Franklin Lanes, NJ, USA) on the upper surface of the polycarbonate membrane inserts (pore size, $8 \mu \mathrm{m}$ ). Following incubation at $37^{\circ} \mathrm{C}$ for $30 \mathrm{~min}$, the Matrigel became solidified and served as the extracellular matrix for cell invasion analysis. A total of $2 \mathrm{ml}$ cells (containing $5 \times 10^{4}$ cells) suspension was added to the upper compartment and $1 \mathrm{ml}$ DMEM + fetal calf serum (Gibco; Thermo Fisher Scientific, Inc.) was added to the lower compartment. After $36 \mathrm{~h}$ of incubation at $37^{\circ} \mathrm{C}$ in $5 \% \mathrm{CO}_{2}$, the cells were allowed to migrate from the Matrigel into the pores of the filters. The membranes were excised from the insert and the cells on the upper surface of the membrane were removed with a cotton bud. The inserts were then fixed in $95 \%$ alcohol for $20 \mathrm{~min}$ at room temperature and stained with toluidine blue for $5 \mathrm{~min}$ at room temperature.

The number of migrated cells was counted using five randomly selected visual fields from the central and peripheral portion of the filters by an inverted microscope at high-power magnification $(4 \times 10 \times 25)$. Each experiment was repeated three times.

MTT assay for proliferation detection. Cell proliferation was measured using the MTT Cell Proliferation and Cytotoxicity Detection kit (Nanjing Keygen Biotech Co., Ltd., Nanjing, China), according to the manufacturer's protocols. Briefly, all groups of cells were placed in 96-well plates (Costar; Corning Incorporated) at a density of $1 \times 104$ cells/well in a humidified atmosphere $\left(37^{\circ} \mathrm{C}, 5 \% \mathrm{CO}_{2}\right)$. After a 24 -h incubation, $10 \mu \mathrm{l}$ MTT labeling reagent was added (final concentration, $0.5 \mathrm{mg} / \mathrm{ml}$ ) to each well and incubated again at $37^{\circ} \mathrm{C}$ with $5 \%$ $\mathrm{CO}_{2}$ for $4 \mathrm{~h}$. Subsequently, $50 \mu \mathrm{l} 1 \mathrm{X}$ MTT was added to each well, followed by incubation at $37^{\circ} \mathrm{C}$ for $4 \mathrm{~h}$. After the medium containing MTT was absorbed, the purple formazan crystals were dissolved in $150 \mu \mathrm{l}$ dimethyl sulfoxide (Sigma-Aldrich; Merck KGaA, Darmstadt, Germany). The absorbance of each well was measured on a microtiter plate reader at a wavelength of $570 \mathrm{~nm}$. Each experiment was repeated three times.

Apoptosis assay. An Annexin V-fluorescein isothiocyanate (FITC)/propidium iodide (PI) kit (catalog no., A0001a; Adlitteram Diagnostic Laboratories, San Diego, CA, USA) was used with a FACSAria ${ }^{\mathrm{TM}}$ flow cytometer (BD Biosciences). Briefly, the harvested cells were washed with PBS at $4^{\circ} \mathrm{C}$ and centrifuged at $300 \mathrm{x}$ g for $5 \mathrm{~min}$ at $4^{\circ} \mathrm{C}$, prior to $100 \mu \mathrm{l}$ Annexin $\mathrm{V}$ labeling reagent being added (10 $\mu \mathrm{l} 10 \mathrm{X}$ binding buffer, $5 \mu \mathrm{l}$ Annexin V-FITC, $85 \mu \mathrm{l}$ distilled water), followed by a 15 -min incubation in the dark. Following the incubation, $10 \mu \mathrm{l}$ PI and $400 \mu \mathrm{l}$ cold $1 \mathrm{X}$ binding buffer were added to each well to dilute the cell suspension. Finally, cell apoptosis was measured using a flow cytometer (FACSAriaTM, CellQuestPro, BD Biosciences). This experiment was repeated three times.

Statistical analysis. All data are presented as the mean \pm standard deviation. Statistical analysis was performed by one-way analysis of variance, followed by the Student-Newman-Keuls method, or two-tailed Student's t-tests. SPSS 13.0 software (SPSS, Inc., Chicago, IL, USA) was used for data analysis. $\mathrm{P}<0.05$ was considered to indicate a statistically significant difference. Statistically significant differences are annotated as ${ }^{*} \mathrm{P}<0.05,{ }^{* *} \mathrm{P}<0.01$ and ${ }^{* * * *} \mathrm{P}<0.001$.

\section{Results}

Expression of VEGF in hilar cholangiocarcinoma lines. The mRNA expression of VEGF in the QBC939 group was higher 
A

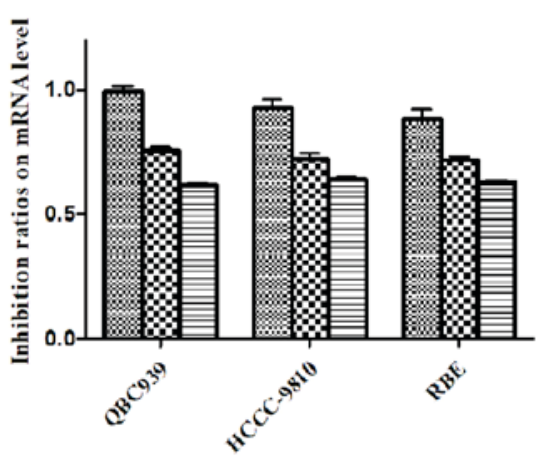

B

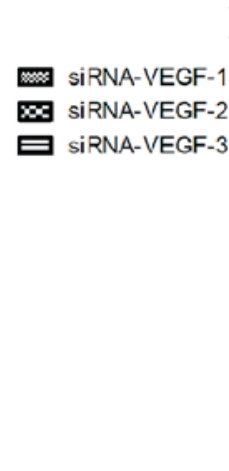

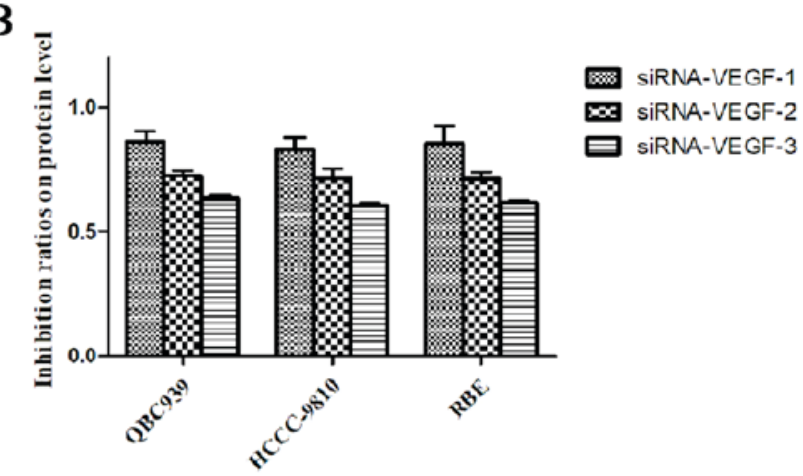

Figure 2. Inhibition ratios of mRNA and protein levels in the three cell lines. (A) The inhibition ratios of mRNA levels in the three cell lines were as follows: siRNA-VEGF-1 (92.21, 93.04 and 88.12\%), siRNA-VEGF-2 (75.66, 72.31 and 71.67\%) and siRNA-VEGF-3 (61.79, 64.0, and 62.98\%). (B) The inhibition ratios of protein levels in the three cell lines were as follows: siRNA-VEGF-1 (86.43, 83.02 and 85.62\%), siRNA-VEGF-2 (72.43; 71.55 and 71.24\%) and siRNA-VEGF-3 $(63.59,60.37$ and $61.93 \%)$, respectively. siRNA-VEGF, vascular endothelial growth factor small interfering RNA.

A

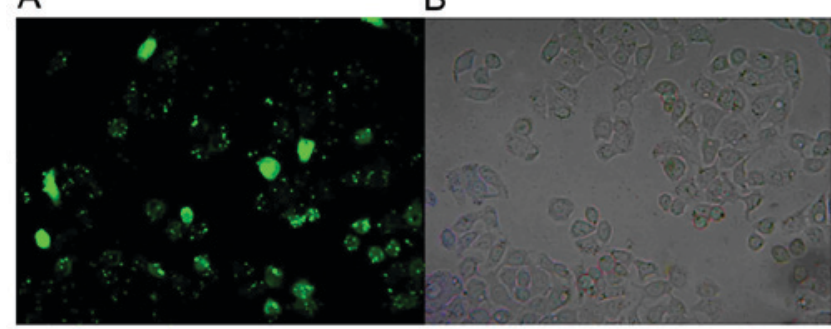

C

D

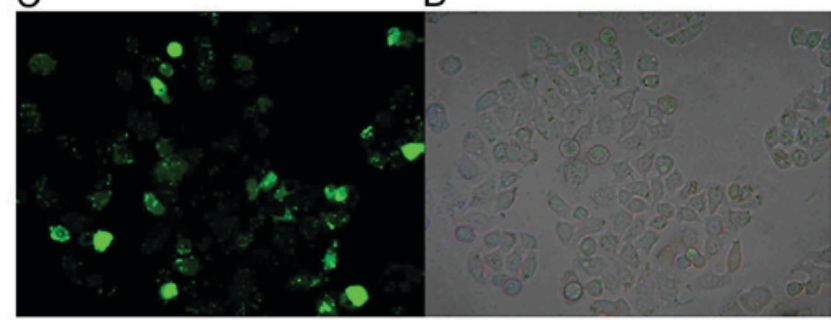

Figure 3. Transfection of cells with siRNA-VEGF-1. The transfection rate in the VEGF-siRNA-1 and negative control groups was $\sim 80 \%$ on average. (A and B) Fluorescent and bright-field images following transfection of siRNA-VEGF-1. (C and D) Fluorescent and bright-field images following transfection with the siRNA-NC. Magnification, 4x10x25. siRNA-VEGF, vascular endothelial growth factor small interfering RNA.

than that in the RBE group and HCCC-9801 groups (16.62 vs. 15.35 and $6.21 ; \mathrm{P}<0.001)$. The same result was also observed at the protein level (Fig. 1). Therefore, the QBC939 cell line was used for further experiments.

Inhibition ratios of $m R N A$ and protein levels. RT-qPCR and western blotting revealed that in the QBC939, HCCC-9810 and RBE cell lines, the expression of VEGF at the mRNA and protein levels in the siRNA-VEGF-1 groups was significantly reduced. The inhibition ratios of mRNA levels in the three cell lines were as follows: siRNA-VEGF-1 (92.21,93.04 and 88.12\%), siRNA-VEGF-2 (75.66, 72.31 and 71.67\%) and siRNA-VEGF-3 $(61.79,64.05$ and $62.98 \%)$. The inhibition ratios of protein levels in the three cell lines were as follows: siRNA-VEGF-1 (86.43, 83.02 and 85.62\%), siRNA-VEGF-2 (72.43, 71.55 and $71.24 \%$ ) and siRNA-VEGF-3 (63.59, 60.37 and $61.93 \%)$. siRNA-VEGF-1 was the most effective at inhibiting VEGF

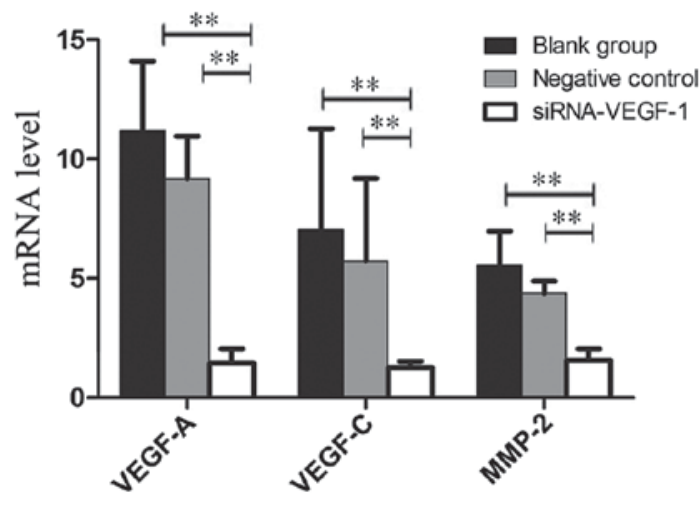

Figure 4. Downregulated expression of VEGF-A, VEGF-C and MMP-2 in QBC939 cells transfected with VEGF-siRNA-1. There was a significant inhibition of VEGF-A, VEGF-C and MMP-2 mRNA levels in the siRNA-VEGF-1 group compared with that in the two control groups. The VEGF-A mRNA levels in the blank control, NC and VEGF-siRNA-1 groups were $11.21 \pm 2.89,9.18 \pm 1.78$ and $1.44 \pm 0.62$, respectively. The inhibition ratio by VEGF-siRNA-1 was $87.12 \%(\mathrm{P}<0.01)$, while the NC group exhibited no significant differences in VEGF-A mRNA levels in QBC939 cells $(\mathrm{P}>0.05)$. Similar outcomes were observed in the VEGF-C and MMP-2 mRNA expression levels, with inhibition ratios of 81.98 and $71.62 \%$, respectively. ${ }^{*} \mathrm{P}<0.05$, ${ }^{* *} \mathrm{P}<0.01$ and ${ }^{* * *} \mathrm{P}<0.001$. VEGF, vascular endothelial growth factor; MMP-2, matrix metalloproteinase 2; siRNA, small interfering RNA; NC, negative control.

expression ( $\mathrm{P}<0.05$, Fig. 2), with no significant differences between the three cell lines being observed $(\mathrm{P}>0.05)$. According to these results, the QBC939 cell line and siRNA-VEGF-1 were selected for further study. The experimental design used siRNA-VEGF-1 and shRNA-VEGF-NC as the treatment and negative control (NC) groups, respectively, whereas cells in the blank control (BC) group were not treated with any siRNA.

Downregulation of VEGF-A, VEGF-C and MMP-2 mRNA expression by siRNA in QBC939 cells. The VEGF-siRNA-1 and scrambled shRNA were transiently transfected into QBC939 cells for $48 \mathrm{~h}$. The transfection rates in the VEGF-siRNA-1 and siRNA-VEGF-NC groups were $\sim 80 \%$ (Fig. 3A-D). After the cells had been passaged once, the transfection rates remained unchanged.

As demonstrated in Fig. 4, a significant reduction in VEGF-A, VEGF-C and MMP-2 mRNA expression was 

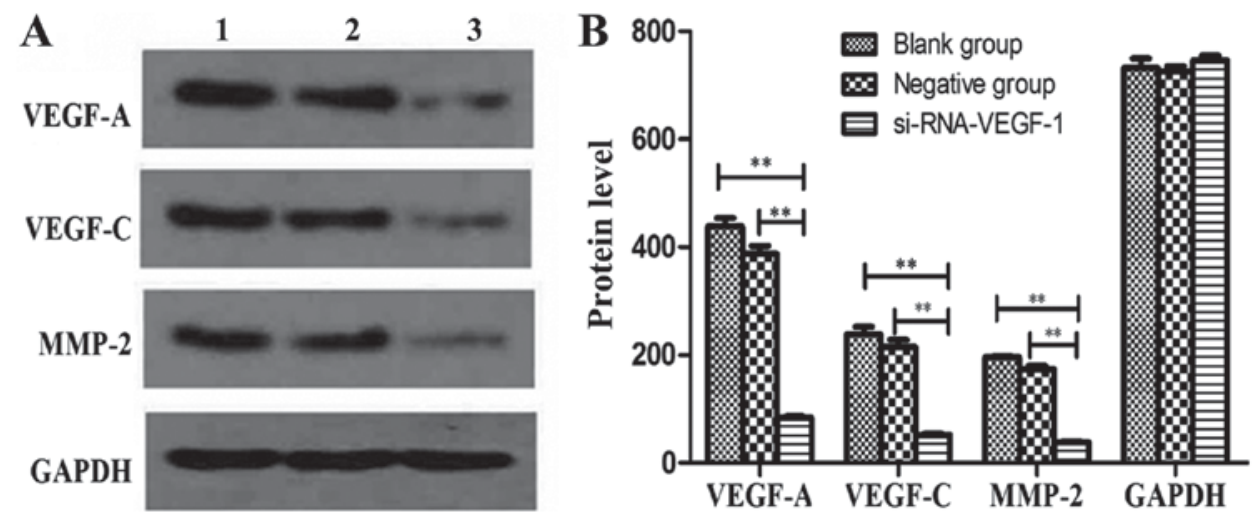

Figure 5. Reduction in VEGF-A, VEGF-C and MMP-2 protein levels in QBC939 cells transfected with VEGF-siRNA-1. (A) The protein levels of VEGF-A, VEGF-C, MMP-2 and GAPDH by western blotting. (B) The integrated optical density value of VEGF-A, VEGF-C, MMP-2 and GAPDH assayed by Gel-pro Analyzer 4.0. The inhibition ratios of the proteins were as follows: $80.85 \%$ for VEGF-A, 77.96\% for VEGF-C and $80.25 \%$ for MMP-2. The differences were significantly greater in the VEGF-siRNA-1 group than in the two control groups $(\mathrm{P}<0.01)$. There was no significant difference between the blank control and negative control groups $(\mathrm{P}>0.05){ }^{* * *} \mathrm{P}<0.01$. VEGF, vascular endothelial growth factor; MMP-2, matrix metalloproteinase 2; siRNA, small interfering RNA.
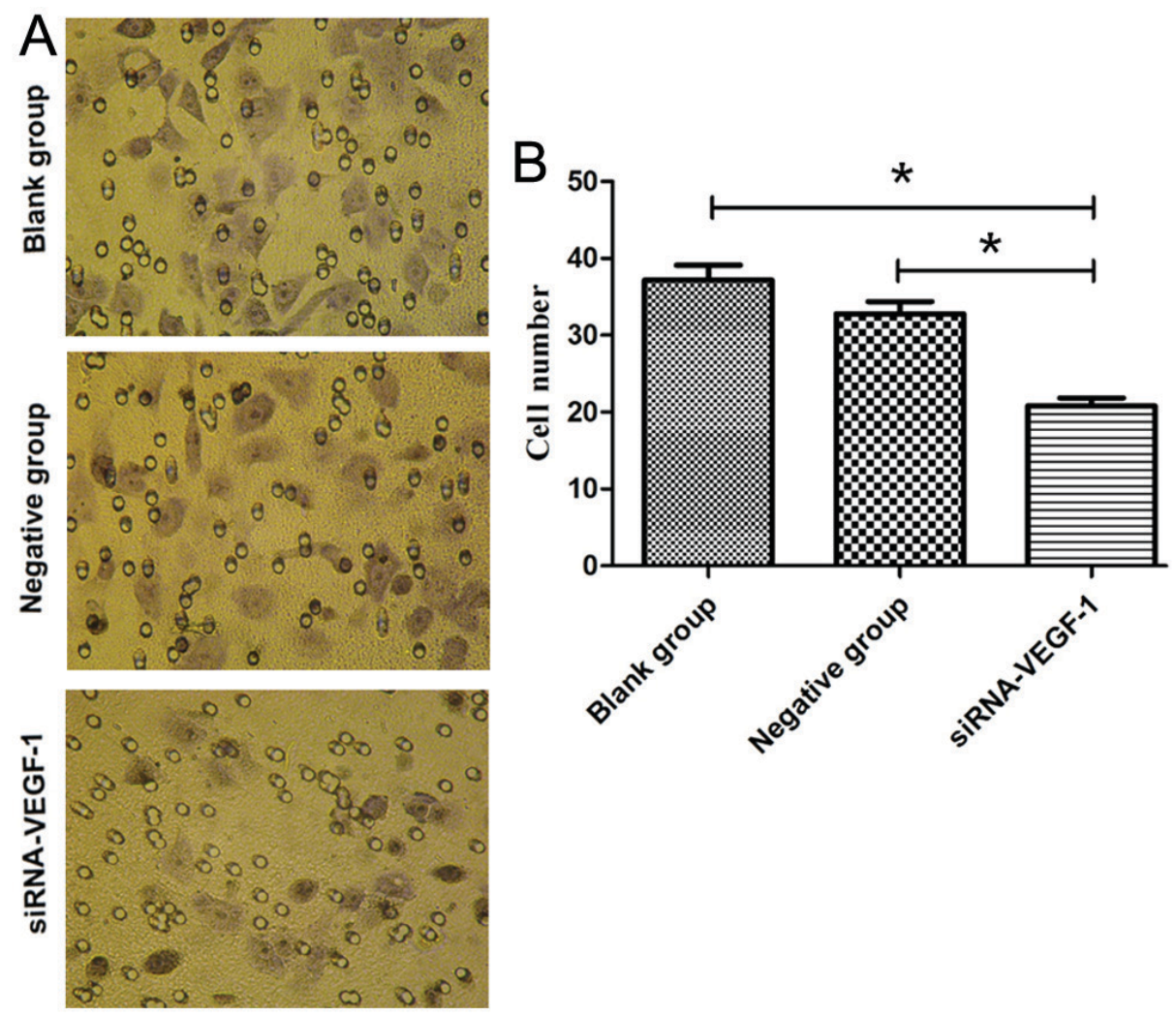

Figure 6. Effects of VEGF-siRNA-1 on QBC939 cell invasion. (A) The migration of QBC939 cells following treatment with VEGF-siRNA, tested by Transwell assays. (B) A representative $x 1,000$ bright field image under an inverted microscope. The number of migrated cells in the VEGF-siRNA group was 20.8 \pm 2.39 , significantly lower than that in the blank control group (37.2 \pm 4.32$)$ and the negative control group $(32.8 \pm 3.56, \mathrm{P}<0.05)$. ${ }^{*} \mathrm{P}<0.05$. VEGF, vascular endothelial growth factor; siRNA, small interfering RNA.

observed in the VEGF-siRNA-1 group compared with the two control groups $48 \mathrm{~h}$ post-transfection. The VEGF-A mRNA levels in the BC, NC and VEGF-siRNA-1 group were $11.21 \pm 2.89,9.18 \pm 1.78$ and $1.44 \pm 0.62$, respectively. The inhibition ratio was $87.12 \%$ by VEGF-siRNA-1 $(\mathrm{P}<0.01)$, while the $\mathrm{NC}$ group had no significant difference in expression of VEGF-A mRNA in QBC939 cells (P>0.05). Similar outcomes were observed in VEGF-C and MMP-2 mRNA expression, with inhibition ratios of 81.98 and $71.62 \%$, respectively.
Downregulation of VEGF-A, VEGF-C and MMP-2 protein levels in QBC939 cells. Levels of the target proteins and GAPDH were analyzed by western blotting, and images were captured using a digital video camera (Fig. 5A). The protein levels of VEGF-A, VEGF-C and MMP-2 were significantly downregulated following siRNA treatment. The inhibition ratio of the proteins varied among the three groups, with ratios of: $80.85 \%$ for VEGF-A, $77.96 \%$ for VEGF-C and $80.25 \%$ for MMP-2. The differences were significantly greater in the 


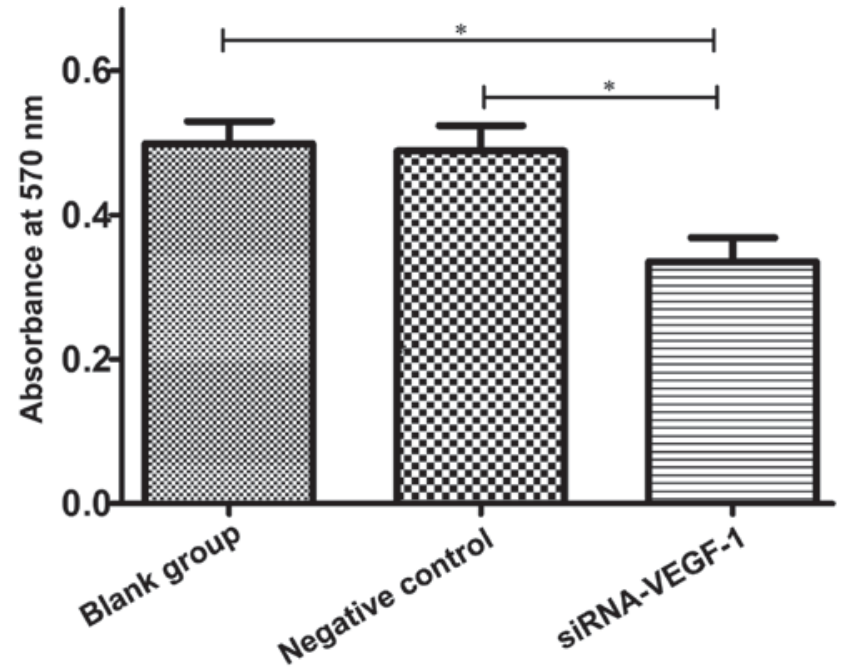

Figure 7. Proliferation inhibition of QBC939 cells transfected with siRNA-VEGF-1. Cell proliferation was significantly reduced in VEGF-siRNA-1-transfected cells $(0.302 \pm 0.004)$ compared with that in the blank $(0.466 \pm 0.0045)$ and negative control groups $(0.455 \pm 0.0026) .{ }^{*} \mathrm{P}<0.05$ VEGF, vascular endothelial growth factor; siRNA, small interfering RNA.

VEGF-siRNA-1 group than in the two control groups $(\mathrm{P}<0.01)$; however, there was no significant difference between the $\mathrm{BC}$ and $\mathrm{NC}$ groups ( $\mathrm{P}>0.05$, Fig. 5B).

Reduced invasion of QBC939 cells. As shown in Fig. 6, for each 1000x field under the inverted microscope, the number of migrated cells in the VEGF-siRNA group was 20.8 \pm 2.39 , significantly lower than that of the BC group (37.2 \pm 4.32$)$ and the $\mathrm{NC}$ group $(32.8 \pm 3.56, \mathrm{P}<0.05)$. In addition, there was no significant difference between the two control groups $(\mathrm{P}>0.05)$.

Reduced proliferation of QBC939 cells. As demonstrated in Fig. 7, the survival rate of the QBC939 cells was measured by the MTT assay in the VEGF-siRNA-1 group and the NC group. Cell proliferation was significantly reduced in VEGF-siRNA-1-transfected cells $(0.302 \pm 0.004)$ compared with the blank $(0.466 \pm 0.0045)$ and negative control groups $(0.455 \pm 0.0026)$. The survival rate in the VEGF-siRNA-1 group was $64.3 \%$, significantly lower than that of the BC group $(100 \%)$ and the NC group $(97.5 \%$; $\mathrm{P}<0.01)$. There was no difference between the $\mathrm{BC}$ and $\mathrm{NC}$ groups $(\mathrm{P}>0.05)$.

Induction of apoptosis by VEGF-siRNA in QBC939 cells. Significant apoptosis was observed in the VEGF-siRNA group compared with that in the two control groups. In the VEGF-siRNA-1 group, the rate of apoptosis of QBC939 cells reached $15.42 \%$, significantly higher than that of the BC group $(2.22 \%)$ and the $\mathrm{NC}$ group $(2.71 \%, \mathrm{P}<0.05)$. However, there was no significant difference between the two controls (P>0.05; Fig. 8).

\section{Discussion}

The majority of patients with hilar cholangiocarcinoma are diagnosed in the late stage of disease and have little chance to undergo surgery, resulting in a poor prognosis (28).
Advances in interventional radiology and endoscopy have enabled non-surgical palliation of this disease; however, the effects are limited $(9,29)$. The majority of patients with hilar cholangiocarcinoma are treated with conventional chemotherapy as the primary treatment of choice. In such clinical settings, an aggressive regimen of chemotherapy may not only fail to improve survival but may also harm the quality of life (28).

These clinical realities provide a strong rationale for the development of novel therapeutic strategies. RNAi techniques have made remarkable progress in in vitro studies over the last decade, in 2002, McCaffrey et al (30) published the first in vivo study for gene silencing by siRNA using mouse models. Subsequently, other studies have demonstrated that highly specific siRNAs have significant effects on the treatment of certain malignant tumors, including gastrointestinal cancer, liver cancer, mammary cancer, prostatic cancer and pancreatic cancer (31-34). The success of those experiments using RNAi gene-silencing techniques caused widespread interest for the development of RNAi-based therapeutics. In light of these unmet clinical requirements, the present study aimed to identify a novel approach to more effectively treat hilar cholangiocarcinomas.

According to the results of the present study, siRNA sequences targeting the VEGF gene were successfully designed and synthesized and siRNA can effectively silence VEGF expression. When VEGF is blocked, VEGF-A, VEGF-C and MMP2 expression at the mRNA and protein levels is inhibited in the QBC939 cell line. There have been a series of studies (35-37) that have established the key role that VEGF serves in the angiogenesis and metastasis of tumors. This function is mediated by members of the VEGF signaling family, including VEGF-A, VEGF-B, VEGF-C, VEGF-D and VEGF-E, as well as the VEGF receptors VEGFR-1 to VEGFR-3 $(10,35,37)$.

In addition, the results of the present study using Transwell and MTT assays revealed that the invasion, migration and proliferation of these tumor cells can be suppressed following VEGF silencing. Results from the apoptosis assay also revealed that the rate of apoptosis was increased in the VEGF-siRNA-1 group. These results can be explained by the combined functions of VEGF-A, VEGF-C and MMP2. VEGF-A is believed to stimulate angiogenesis by activating endothelial cell proliferation via the intracellular tyrosine phosphorylation pathway through a paracrine or autocrine mechanism. In addition, VEGF-A acts as a major anti-apoptotic factor for endothelial cells observed in immature, newly formed blood vessels (38-40). Therefore, blocking VEGF-A expression should effectively suppress intra-tumoral angiogenesis, vascular permeability and tumor growth. VEGF-C is regarded as a crucial factor for regulating lymphogenesis, acting via VEGFR-3. Certain studies have also demonstrated that there is a correlation between tumor VEGF-C expression and lymph node metastasis (41-43). This suggested that blocking the expression of VEGF-C in the tumor cells can relieve lymphatic invasion and lymph node metastasis. MMP2 acts as a gelatinase, which is a major factor for homeostatic regulation of the extracellular environment. MMP2 can promote cancer progression, as well as allow cancer cell invasion, migration and metastasis by degrading the extracellular matrix and regulating the function of bioactive molecules by proteolytic processing (44-46). 

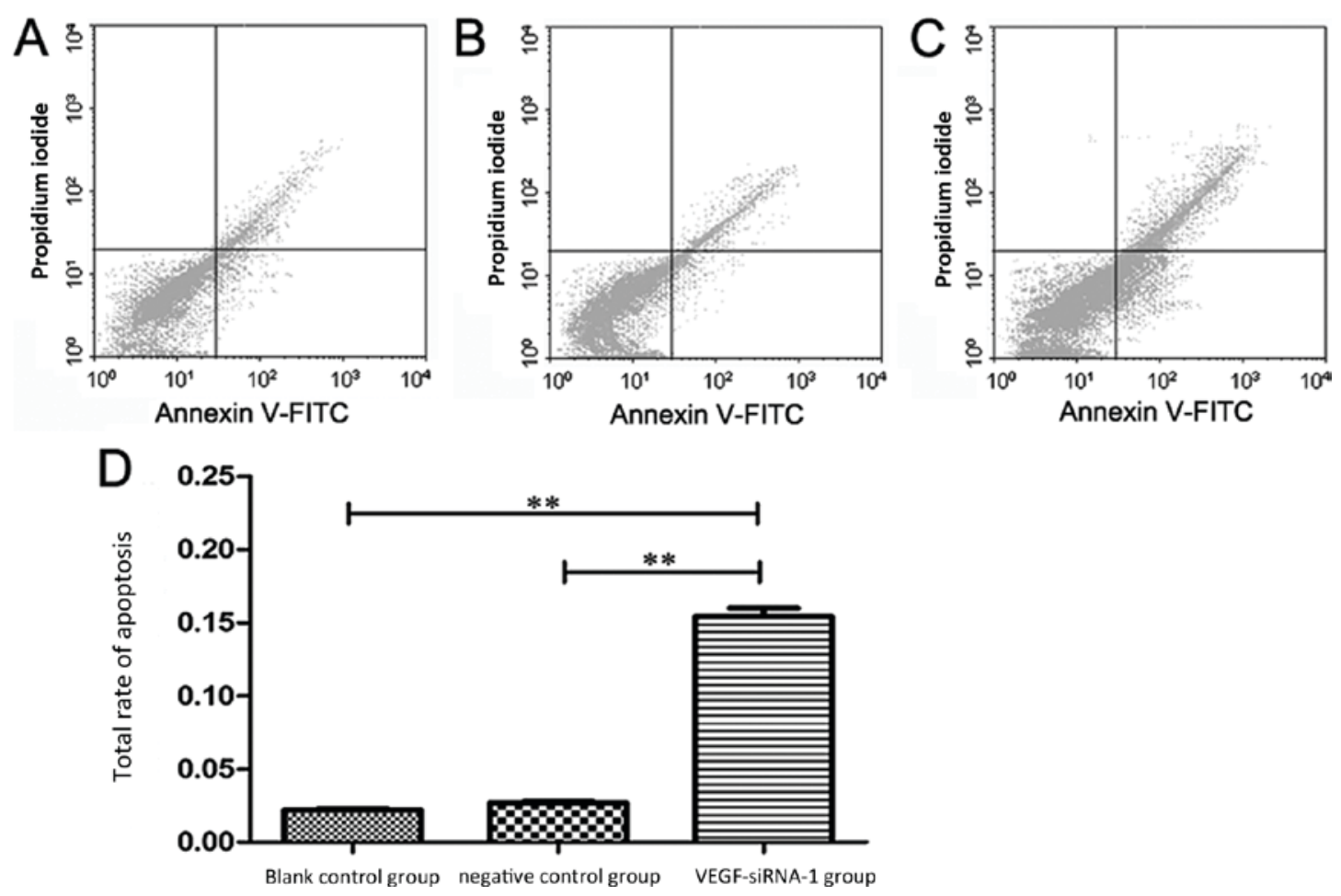

Figure 8. Rate of apoptosis in QBC939 cells transfected with VEGF-siRNA-1. The total rate of apoptosis was the rate from the right upper quadrant added to that of the right lower quadrant. (A) The total rate of apoptosis in the blank control group: $0.78+1.44=2.22 \%$. (B) The total rate of apoptosis in the negative control group: $0.84+1.87=2.71 \%$. (C) The total rate of apoptosis in the VEGF-siRNA-1 group: $7.29+8.13=15.42 \%$. (D) The column chart of apoptosis percentage in the three groups. ${ }^{* *} \mathrm{P}<0.01$. VEGF, vascular endothelial growth factor; siRNA, small interfering RNA.

In summary, VEGF-A, VEGF-C and MMP2 serve key roles in the angiogenesis, proliferation and metastasis of hilar cholangiocarcinoma. These observations suggested that an RNAi approach targeting VEGF maybe an effective therapeutic strategy for treating hilar cholangiocarcinoma. In addition, detection of the serum levels of VEGF-A, VEGF-C and MMP2 may be a useful and significant prognostic indicator, although this possibility requires substantial future research. Furthermore, one limitation of the present study is that it only incorporated a single cell line. Future studies should include two or three different cell lines in order to further verify the conclusions of the present study.

\section{Acknowledgements}

Not applicable.

\section{Funding}

The present study was supported by the National Natural Science Foundation for Youths (grant no. 81702670).

\section{Availability of data and materials}

The datasets used or analyzed during the current study are available from the corresponding author on reasonable request.

\section{Authors' contributions}

$\mathrm{KX}$ and HHT conceived and designed the study. KX and ZO performed the experiments. KX wrote the paper. HHT analyzed the data, and reviewed and edited the manuscript. All authors read and approved the manuscript.

\section{Ethics statement and consent to participate}

Not applicable.

\section{Consent for publication}

Not applicable.

\section{Competing interests}

The authors declare that they have no competing interests.

\section{References}

1. Blechacz B and Gores GJ: Cholangiocarcinoma: Advances in pathogenesis, diagnosis, and treatment. Hepatology 48: 308-321, 2008.

2. Ito F, Cho CS, Rikkers LF and Weber SM: Hilar cholangiocarcinoma: Current management. Ann Surg 250: 210-218, 2009.

3. Blendis L and Halpern Z: An increasing incidence of cholangiocarcinoma: Why? Gastroenterology 127: 1008-1009, 2004.

4. Shin HR, Oh JK, Masuyer E, Curado MP, Bouvard V, Fang YY, Wiangnon S, Sripa B and Hong ST: Epidemiology of cholangiocarcinoma: An update focusing on risk factors. Cancer Sci 101: 579-585, 2010.

5. Jemal A, Bray F, Center MM, Ferlay J, Ward E and Forman D: Global Cancer Statistics. CA Cancer J Clin 61: 69-90, 2011.

6 . Feige JJ: Tumor angiogenesis: Recent progress and remaining challenges. Bull Cancer 97: 1305-1310, 2010 (In French).

7. Tang D, Nagano H, Yamamoto H, Wada H, Nakamura M, Kondo M, Ota H, Yoshioka S, Kato H, Damdinsuren B, et al: Angiogenesis in cholangiocellular carcinoma: Expression of vascular endothelial growth factor, angiopoietin- $1 / 2$, thrombospondin-1 and clinicopathological significance. Oncol Rep 15: 525-532, 2006. 
8. Thelen A, Scholz A, Weichert W, Wiedenmann B, Neuhaus P, Gessner R, Benckert C and Jonas S: Tumor-associated angiogenesis and lymphangiogenesis correlate with progression of intrahepatic cholangiocarcinoma. Am J Gastroenterol 105: 1123-1132, 2010.

9. Halper J: Growth factors as active participants in carcinogenesis: A perspective. Vet Pathol 47: 77-97, 2010.

10. Appelmann I, Liersch R, Kessler T, Mesters RM and Berdel WE: Angiogenesis inhibition in cancer therapy: Platelet-derived growth factor (PDGF) and vascular endothelial growth factor (VEGF) and their receptors: Biological functions and role in malignancy. Recent Results Cancer Res 180: 51-81, 2010.

11. Zhou J and Tang HH: Significance and expression of microvessel density, vascular endothelial growth factor and matrix metalloproteinases 2 in cholangiocarcinoma. Chin J Gen Surg 12: 122-124, 2003 (In Chinese).

12. Das S, Ladell DS, Podgrabinska S, Ponomarev V, Nagi C, Fallon JT and Skobe M: Vascular endothelial growth factor-C induces lymphangitic carcinomatosis, an extremely aggressive form of lung metastases. Cancer Res 70: 1814-1824, 2010.

13. Achen MG and Stacker SA: Molecular control of lymphatic metastasis. Ann NY Acad Sci 1131: 225-234, 2008.

14. Vázquez-Vega S, Contreras-Paredes A, Lizano-Soberón M, Amador-Molina A, García-Carrancá A, Sánchez-Suárez LP and Benítez-Bribiesca L: RNA interference (RNAi) and its therapeutic potential in cancer. Rev Invest Clin 62: 81-90, 2010 (In Spanish).

15. Farazi TA, Spitzer JI, Morozov P and Tuschl T: miRNAs in human cancer. J Pathol 223: 102-115, 2011.

16. Lares MR, Rossi JJ and Ouellet DL: RNAi and small interfering RNAs in human disease therapeutic applications. Trends Biotechnol 28: 570-579, 2010.

17. Sui G, Soohoo C, Affar el B, Gay F, Shi Y, Forrester WC and Shi Y: A DNA vector based RNAi technology to suppress gene expression in mammalian cells. Proc Natl Acad Sci USA 99: 5515-5520, 2002.

18. Yu JY, DeRuiter SL and Turner DL: RNA interfering by expression of short interfering RNAs and hairpin RNAs in mammalian cells. Proc Natl Acad Sci USA 99: 6047-6052, 2002.

19. McManus MT and Sharp PA: Gene silencing in mammals by small interfering RNAs. Nat Rev Genet 3: 737-747, 2002.

20. Macedo F, Ladeira K, Longatto-Filho A and Martins SF: gastric cancer and angiogenesis: Is VEGF a useful biomarker to assess progression and remission? J Gastric Cancer 17: 1-10, 2017.

21. Chen J, Wu H, Han D and Xie C: Using anti-VEGF McAb and magnetic nanoparticles as double-targeting vector for the radioimmunotherapy of liver cancer. Cancer Lett 231: 169-175, 2004.

22. Queiroga FL, Pires I, Parente M, Gregório H and Lopes CS: COX-2 over-expression correlates with VEGF and tumour angiogenesis in canine mammary cancer. Vet J 189: 77-82, 2011.

23. Botelho F, Pina F and Lunet N: VEGF and prostatic cancer: A systematic review. Eur J Cancer Prev 19: 385-392, 2010.

24. Eibl G, Bruemmer D, Okada Y, Duffy JP, Law RE, Reber HA and Hines OJ: PGE (2) is generated by specific COX-2 activity and increases VEGF production in COX-2-expressing human pancreatic cancer cells. Biochem Biophys Res Commun 306 887-897, 2003

25. Shah MA: The development of bevacizumab in noncolorectal gastrointestinal malignancies: Gastroesophageal, pancreatic, and hepatocellular carcinoma. Clin Adv Hematol Oncol 12: 239-246, 2014.

26. Bhardwaj A, Kaur J, Wuest F and Knaus EE: Fluorophore-labeled cyclooxygenase-2 inhibitors for the imaging of cyclooxygenase-2 overexpression in cancer: Synthesis and biological studies. ChemMedChem 9: 109-116, 240, 2014.

27. Livak KJ and Schmittgen TD: Analysis of relative gene expression data using real-time quantitative PCR and the 2(-Delta Delta C(T)) method. Methods 25: 402-408, 2001.

28. Mansour JC, Aloia TA, Crane CH, Heimbach JK, Nagino M and Vauthey JN: Hilar cholangiocarcinoma: Expert consensus statement. HPB (Oxford) 17: 691-699, 2015.
29. Tsao JI, Nimura Y, Kamiya J, Hayakawa N, Kondo S, Nagino M, Miyachi M, Kanai M, Uesaka K, Oda K, et al: Management of Hilar Cholangiocarcinoma: Comparison of an American and a Japanese experience. Ann Surg 232: 166-174, 2000.

30. McCaffrey AP, Meuse L, Pham TT, Conklin DS, Hannon GJ and Kay MA: RNA interference in adult mice. Nature 418: 38-39, 2002.

31. Petrocca F and Lieberman J: Promise and challenge of RNA interference-based therapy for cancer. J Clin Oncol 29: 747-754, 2011.

32. Addepalli MK, Ray KB, Kumar B, Ramnath RL, Chile S and Rao H: RNAi-mediated knockdown of AURKB and EGFR shows enhanced therapeutic efficacy in prostate tumor regression. Gene Ther 17: 352-359, 2010.

33. Cuevas EP, Escribano O, Monserrat J, Martínez-Botas J, Sánchez MG, Chiloeches A, Hernández-Breijo B, Sánchez-Alonso V, Román ID, Fernández-Moreno MD and Guijarro LG: RNAi-mediated silencing of insulin receptor substrate-4 enhances actinomycin D- and tumor necrosis factor-alpha-induced cell death in hepatocarcinoma cancer cell lines. J Cell Biochem 108: 1292-1301, 2009.

34. Varnholt H: The role of microRNAs in primary liver cancer. Ann Hepatol 7: 104-113, 2008.

35. Holash J, Davis S, Papadopoulos N, Croll SD, Ho L, Russell M, Boland P, Leidich R, Hylton D, Burova E, et al: VEGF-Trap: A VEGF blocker with potent antitumor effects. Proc Natl Acad Sci USA 99: 11393-11398, 2002.

36. Ylä-Herttuala S: Gene therapy with vascular endothelial growth factors. Biochem Soc Trans 37: 1198-1200, 2009.

37. Grünewald FS, Prota AE, Giese A and Ballmer-Hofer K: Structure-function analysis of VEGF receptor activation and the role of coreceptors in angiogenic signaling. Biochim Biophys Acta 1804: 567-580, 2010

38. Geva R, Prenen H, Topal B, Aerts R, Vannoote J and Van Cutsem E: Biologic modulation of chemotherapy in patients with hepatic colorectal metastases: The role of anti-VEGF and anti-EGFR antibodies. J Surg Oncol 102: 937-945, 2010.

39. Vermeulen PB, van Golen KL and Dirix LY: Angiogenesis, lymphangiogenesis, growth pattern, and tumor emboli in inflammatory breast cancer: A review of the current knowledge. Cancer 116 (Suppl 11): S2748-S2754, 2010.

40. Winder T and Lenz HJ: Vascular endothelial growth factor and epidermal growth factor signaling pathways as therapeutic targets for colorectal cancer. Gastroenterology 138: 2163-2176, 2010.

41. Kluger MS and Colegio OR: Lymphangiogenesis linked to VEGF-C from tumor-associated macrophages: Accomplices to metastasis by cutaneous squamous cell carcinoma? J Invest Dermatol 131: 17-19, 2011.

42. Beeghly-Fadiel A, Shu XO, Lu W, Long J, Cai Q, Xiang YB, Zheng Y, Zhao Z, Gu K, Gao YT and Zheng W: Genetic variation in VEGF family genes and breast cancer risk: A report from the Shanghai Breast Cancer Genetics Study. Cancer Epidemiol Biomark Prev 20: 33-41, 2011.

43. Nakatani H, Akimori T, Takezaki Y and Hanazaki K: Vascular endothelial growth factors and their receptors in the novel human cell line, HN-Eso-1, established from esophageal spindle cell carcinoma. J Med Invest 57: 232-236, 2010.

44. Park KS, Kim SJ, Kim KH and Kim JC: Clinical characteristics of TIMP2, MMP2, and MMP9 gene polymorphisms in colorectal cancer. J Gastroenterol Hepatol 26: 391-397, 2011.

45. Overall CM and Dean RA: Degradomics: Systems biology of the protease web. Pleiotropic roles of MMPs in cancer. Cancer Metastasis Rev 25: 69-75, 2006.

46. Gialeli C, Theocharis AD and Karamanos NK: Roles of matrix metalloproteinases in cancer progression and their pharmacological targeting. FEBS J 278: 16-27, 2011. 